\title{
Sarcopenic Obesity in the Elderly
}

\author{
Michael AB Naafs* \\ Dutch Internist-Endocrinologist, Health Consultant at Naafs International Health Consultancy, Netherlands
}

Submission: November 27, 2017; Published: December 05, 2017

*Corresponding author: Michael AB Naafs, Dutch Internist-Endocrinologist, Health Consultant at Naafs International Health Consultancy, Netherlands

Abstract

In this mini-review pathophysiology, comorbidities, diagnosis and management of sarcopenic obesity (SO) are discussed. SO is a high risk geriatric syndrome more associated with osteoarthritis (OA), falls, dementia and increased cardiometabolic risk profiles than obesity alone. Decreased physical activity, low-grade chronic inflammation, oxidative stress and insulin resistance all of which being by-products of obesity and the aging process are involved. Easy diagnostic tools are not yet available and sophisticated DXA and SPECT scans are not always feasible. Prevention and resistance exercise programs combined with protein supplementation are the cornerstone of SO management. When male SO patients can be easily identified, treatment with selective androgen receptor modulators (SARMs) might be considered in the near future.

\section{Introduction}

The prevalence of obesity in combination with sarcopenia, the age-related loss of muscle mass and strength or physical function, is increasing in adults aged 65 and older. A major subset of adults over the age 65 is now classified as having sarcopenic obesity, a high risk geriatric syndrome. Moreover, recent estimates suggest that $37 \%$ of U.S. adults aged 65 year and older are obese [1]. The specific criteria for defining sarcopenic obesity (SO) are somewhat arbitrary and depend on the study cited [2]. Thus, the prevalence of SO varies from $4 \%$ to $84 \%$ in men and from $4 \%$ to $94 \%$ in women [3]. Older adults with SO have higher risks of mobility disability, cardiometabolic disease and mortality [4]. In this mini-review pathophysiology, comorbidities, diagnosis and management of $\mathrm{SO}$ are discussed.

\section{Pathophysiology SO}

Human aging is associated with a progressive decline of skeletal muscle mass. Several studies have suggested that muscle mass decreases by approximately $6 \%$ per decade after midlife [5]. Lower muscle mass results in decreased muscle strength [6]. There are significant differences among individuals in peak muscle mass, the age at which muscle loss starts, and the amount of muscle that is lost over time [7]. At the cellular level sarcopenia is accompanied by a loss of innervation and adaptive changes in the proportion of slow and fast motor units, as well as in the crosssectional area of muscle fibers [8]. Several mechanisms have been linked to the development of sarcopenia [9]. Most mechanisms are also associated with visceral obesity, leading to a vicious circle of interacting risk factors. Insulin resistance plays an important role in obesity and results in muscle fiber atrophy and mitochondrial dysfunction $[9,10]$. Age-related changes in hormones play a pivotal role and affect the anabolic and catabolic processes in skeletal muscle [11,12]. Reduced androgen and estrogen levels decrease muscle mass and strength [12]. In addition, sarcopenia is an inflammatory state that is driven by proinflammatory cytokines and oxidative stress [13]. Oxidative stress modulates the expression of transcription factors, such as nuclear factor-kappa B (NF-kB), which enhances proteolytic pathways and increases the production of proinflammatory cytokines [14]. Tumor necrosis factor-alpha (TNF-alpha) impairs protein synthesis in skeletal muscle by altering translation initiation, which may contribute to sarcopenia [15]. Higher levels of interleukin-6 (IL-6) and C-reactive protein (CRP) are associated with a greater decline in muscle strength [16]. Myostatin (growth differentiation factor 8) inhibits muscle cell growth and differentiation and could be a potential mediator of sarcopenia [17].

Excess nutrient availability and tissue delivery, particularly saturated fat and glucose further contribute to the cluster of insulin resistance, inflammation and oxidative stress that occur in obesity. Resulting adipose tissue dysfunction develops in response to the enhanced demand for lipid storage [18-20]. These changes may result in an "anabolic resistance state" to nutrients where the muscle protein synthesis from nutrients is blunted [2124]. Mtochondrial changes are observed in obese skeletal muscle until late stages $[25,26]$. Their onset may however exacerbate oxidative stress and related metabolic cascades leading to insulin resistance and catabolism. Potential reduction in ATP production may also result in low muscle strength and endurance capacity $[25,26]$. Stem cell dysfunction leads to functionally altered muscle stem cells that may undergo adipocyte differentiation and accompanied fat accumulation [27-29]. Low physical contributes to a positive energy balance [26]. Progressive reduction of physical activity is further observed with disease progression due 
to worsening obesity and musculoskeletal disorders with direct negative impact on muscle protein turnover and muscle oxidative and performance capacity $[30,31]$.

\section{SO and comorbidities}

\section{Osteoarthritis}

Individuals with osteoarthritis (OA) may exhibit a higher prevalence of SO compared with rheumatoid arthritis (RA)[32]. Misra et al. studied a large cohort from the Multicenter Osteoarthritis (MOST) Study, a longitudinal cohort of individuals with or at risk for knee OA. Based on body composition from whole body Dual Energy X-Ray (DXA) subjects were categorized as obese, sarcopenic obese (SO), sarcopenic and non-sarcopenic obese.

Among 1633 subjects with radiographic knee OA at baseline, significant increased risk of incident radiographic knee OA was found among obese (women RR 2,29;95\%CI 1,64-3,20;men RR 1,$73 ; 95 \%$ CI 1,08-2,78) and SO women (RR 1,91;95\%CI 1,733,11 ) but not men ( RR 1,74;95\% CI 0,68-4,46) .Sarcopenia was not associated with knee OA risk (women RR 0,96;95\% CI 0,341,30 ). It was concluded that in this large cohort population, body composition based obesity and SO but not sarcopenia was associated with knee OA risk. Weight loss strategies for knee OA should focus on obesity and SO [33].

Sarcopenia obesity (SO) results in more physical disability than sarcopenia alone or obesity alone and has been strongly implicated in both risk of OA and frailty [34-36]. Total joint arthroplasty (TJA) in adults with obesity is associated with increased surgical risk and prolonged recovery. SO is associated with higher infection rates, poorer function and slower recovery in other clinical populations, but not thoroughly investigated in osteoarthritis [37].

\section{Cardiometabolic complications of SO}

Ma et al. analyzed a cohort of the Framingham Heart Study's Offspring and Omni 1 cohorts for mid-adulthood cardiometabolic risk profiles in patients with SO [38]. Utilizing BMI and sex-specific 24h urinary creatinine excretion,1019 participants from the Framingham cohorts were categorized as non-sarcopenia non obese (NSNO); non-obese sarcopenia, non-SO and SO. Cardiometabolic risk factors were quantified by standard laboratory assessment cross-sectionally and 10,20 and 30 years before SO assessment. NSNO, sarcopenia, obesity and SO accounted for $30,0 \%, 39,6 \%, 20,0 \%$ and $10,4 \%$ of study participants, respectively. Cross-sectionally, participants with SO had a higher proportion of hypertension, metabolic syndrome and type 2 diabetes than those with NSNO or sarcopenia (all $\mathrm{p}<0,03$ ) Similar patterns were observed retrospectively at 10,20 and 30 years. Compared with NSNO or sarcopenia SO was associated with a higher prevalence of type 2 diabetes at 10 years and hypertension and metabolic syndrome at all three points before baseline (all $\mathrm{p}<0,03$ ). Individuals with SO had more type 2 diabetes than those with obesity alone at baseline and 10 years prior (all $\mathrm{p}<0,001$ ). The authors conclude that adults with SO had more adverse midlife cardiometabolic risks, particularly diabetes 10 years earlier.

\section{Falls and SO}

Pasco et al. examined the association between falls and SO, among elderly individuals in the population [39]. Participants were 353 men and 245 women, aged 65-98 years of the Geelong Osteoporosis Study. Body fat and lean body mass were measured using dual energy X-ray absorptiometry (DXA). Body fat mass was expressed as a percentage of weight (\%BF) and appendicular lean mass was adjusted for height (rALM, kg/m2). Poor physical performance was assessed using the timed up \& go (TUG) test. Sarcopenic obesity referred to low-rALM (T score $<1$ ), poor physical performance (TUG> 10s) and obesity ( $\% \mathrm{BF}>25 \%$ for men,35\% for women) Fallers were identified by self-report as having had at least one fall in the previous 12 months. Associations between SO and falls were determined using logistic regression after adjusting for age and sex. In total 219 (36,6\%) had lower rALM,205(34,2\%) had poor physical performance,466 $(77,9 \%)$ were obese and $69(11,5 \%)$ had So. There were 170 (28,4\%) fallers. Falls were more common for those with os than without $(28(40,6 \%)$ vs $42(26,8 \%) ; \mathrm{p}=0,017)$. The like li hoof of falls in association with SO were: SO,OR=1,65 (95\%CI 0,96-2,85), sarcopenia, OR=1,52 $(0,93-2,47)$,poor physical performance and obesity, $O R=1,74$ $(1,16-2,61)$,low $\quad$ r-ALM,OR=1,41 $\quad(0,96-2,06)$,poor physical performance, OR=1,88 $(1,26-2,80)$,obesity $\mathrm{OR}=0,88(0,57-1,35)$. The authors conclude that while obesity per se was not associated with falls there was an increased risk of falls in SO individuals that was of borderline statistical significance and appeared largely a consequence of poor physical performance [39].

\section{SO and dementia}

Sarcopenia and obesity both negatively impact health including cognitive function. Their coexcistense however, can pose an even higher threat likely surpassing their individual effects. Tolea et al assessed the relationship of SO with performance on global-and subdomain-specific tests of cognition [40]. The study was a cross-sectional analysis of data from a series of communitybased aging and memory studies $(n=353)$ with an average age of 69 years with a clinical visit, valid cognitive (Montreal Cognition Assessment) test, functional (grip strength, chair stands) and body composition measurements [40]. The authors found consistent evidence to link SO to poor global cognitive performance in community-dwelling older adults. This effect is best captured by its sarcopenia component with obesity likely having an additive effect. Several mechanisms may explain the obesity-cognitive dysfunction link including decreased participation in physical activity, low-grade chronic inflammation, oxidative stress and insulin resistance all of which being by-products of the aging process [41]. The authors conclude that sarcopenia alone and in combination with SO can be used in clinical practice as indicators of probable cognitive impairment. At risk older adults may benefit from programs addressing loss of cognitive function by maintaining and improving strength and preventing obesity [40]. 


\section{SO diagnosis}

The current definitions of SO combine sarcopenia as defined through variable criteria in the presence of obesity as defined as BMI $>30 \mathrm{~kg} / \mathrm{m} 2$. Simple anthropometric measurements in obese individuals may be biased by confounding adipose depots. Radiological methods that include nuclear magnetic resonance spectroscopy CT scans (SPECT) or dual X-ray absorptiometry have been considered most accurate but are not always available and feasible in this older population. Bio-electrical impedance analysis has been mentioned as an acceptable compromise. Functional measures are heterogenous and include hand-grip, knee-extensor strength and various moblity measurements involving postural and walking tests [42-44]. At this moment there is obviously no ideal methodology to achieve simultaneously maximal precision, safety, and routine applicability.

\section{Management SO}

Management strategies for obesity commonly favor diet changes and aerobic ecercise in order to reduce levels of body fat. However, this approach doesn't address the loss of muscle mass that may occur during weight loss and contribute to sarcopenia. It is of critical importance that management strategies focus on maintenance or accretion of muscle mass as well as fat loss in order to maintain strength, function and resting metabolic rate (RMR). Resistance exercise in combination with protein supplementation, prescribed by a dietician, can achieve these goals. In this way an 8 week resistance exercise program with protein supplementation can improve muscle mass significantly in even frail old men and women [45]. Any person starting a resistant exercise program should have at least a dietary protein intake of $1.5 \mathrm{~g} / \mathrm{kg}$. This is nearly twice the recommended daily amount (RDA) of $0,8 \mathrm{~g} / \mathrm{kg}$ per day by the Food and Nutrition Board of the U.S. When male SO patients can be identified routinely in an easy manner, treatment with selective androgen receptor modulators (SARMs) might be considered in the near future [46].

\section{Conclusion}

Sarcopenic obesity (SO) is definitely a high risk geriatric syndrome. Decreased physical activity, low grade chronic inflammation, oxidative stress and insulin resistance all of which being by-products of obesity and the aging process are involved [41]. It is obvious SO is more associated with osteoarthritis (OA), falls, dementia and increased cardiometabolic risk profiles than obesity alone. Prevention, resistance exercise programs combined with dietary protein supplementation are the cornerstone of SO management [45]. Ideal easy routine diagnostic tools are not yet available. Radiological techniques measuring total body composition are most accurate but not always present or feasible in the elderly. When male SO patients can be easily identified, treatment with selective androgen receptor modulators (SARMs) might be considered in the near future [46].

\section{References}

1. Mather M (2016) Fact sheet aging in the United States; Population Reference Bureau.
2. Sakuma K, Yamagchi A (2016) Current concepts and therapeutic strategy in sarcopenic obesity.

3. Goisser S, Kemmler W, Porzel S (2015) Sarcopenic obesity and complex interventions with nutrition and exercise in community-dwelling older persons-a narrative review. Clin Interv Aging 10: 1267-1282.

4. Bouchonville MF, Villareal DT (2013) Sarcopenic obesity-How Do We Treat It? Curr Opin Endocrinol. Diabetes Obes 20(5): 412-419.

5. Janssen I (2010) Evolution of sarcopenia research. Appl Physiol Nutr Metab 35(5): 707-712.

6. Newman AB, Haggerty CL, Good Paster B (2003) Strength and muscle quality in a well -functioning cohort of older adults; the Health, Aging and Body Composition Study. J Am Geriatr Soc 51(3): 323-30.

7. Janssen I (2011) The epidemiology of sarcopenia. Clin Geriatr Med 27(3): 355-363.

8. Lang T, Streeper T, Cawthon P (2010) Sarcopenia, etiology, clinical consequences, intervention and assessment. Osteoporosis Int 21(4): 543-559.

9. Cruz Jentoft AJ, Bayens JP, Bauer JM (2010) Sarcopenia: European concensus on definition and diagnosis; Report of the European Working Group on Sarcopenia in Older People. Age Ageing 39(4): 412423.

10. Abbatecole AM, Paollisso G, Fatoretti P (2011) Discovering pathways of sarcopenia in older adults; a role to insulin resistance of mitochondria dysfuction. J Nutr Health Aging 15(10): 890-895.

11. Choi KM (2013) Sarcopenia and sarcopenic obesity. Endocrinol Metab (Seoul) 28(2): 86-89.

12. Wang G, Bal L (2012) Sarcopenia in the elderly; basic and clinical issues. Geriatr. Gerontol Int 12(3): 388-396.

13. Jensen $\mathrm{Gl}$ (2008) Inflammation: roles in aging and sarcopenia. JPEN J Patenter Enteral Nutr 32(6): 656-659.

14. Meng SJ, Yu LJ (2010) Oxidative stress, molecular inflammation and sarcopenia. Int J Mol Sci 11(4): 1509-1526.

15. Lang CH, Frost RA, Nairn AC (2002) TNF-alpha impairs heart and skeletal mechanisms protein synthesis by altering translation initiation. Am J Physiol Endocrinol Metab 28(2): E336-47.

16. Schaap LA, Pluym SM, Deeg DJ (2006) Inflammatory markers and loss of muscle mass (sarcopenia) and strength. Am J Med 119(6): 526e917.

17. White TA, Le Brasseur Nk (2014) Myostatin and sarcopenia: opportunities and challenges; a mini-review. Gerontolgy 60(4): 289293.

18. Barazzoni R, Zanetti M, Gorlan Cappellori G (2012) Fatty acids acutely enhance insulin-induced oxidative stress and cause insulin resistance by increasing mitochondrial reactive species (ROS) generation and nuclear factor-kB inhibitor (LkB)- (NF-kB) activation in rat muscle, in the absence of mitochondrial dysfunction. Diabetologia 55: 733-72.

19. Barazzoni R, Eutz NE, NE, Biolo G (2017) Carbohydrates and insulin resistance in clinical nutrition; recommendations from the ESPEN expert group. Clin Nutr 36(2): 355-363.

20. Czech MP (2017) Insulin action and resistance in obesity and type 2 diabetes. Nat Med 23(7): 804-814.

21. Guillet C, Delcourt I, Rance M (2009) Changes in basal and insulin and amino acid response of whole body and skeletal muscle protein in obese men J. Clin Endocrinol Metab 94(8): 3044-3050.

22. Murton AJ, Marimuthu K, Mallinson JE (2015) Obesity appears to be associated with altered muscle protein synthetic and breakdown responses to increased delivery in older men, but not reduced muscle mass or contractile function. Diabetes 64(9): 3160-3071. 
23. Beals JW, Sukiennik RA, Nalabelli J (2016) Anabolic sensitivity of postprandial muscle protein synthesis to the ingestion of a proteindense food is reduced in overweight and obese young adults. Am J Clin Nutr 104(4): 1014-1022.

24. Smeuninx B, McKendry J, Wilson D (2017) Age-related anabolic resistance of myofibrillar protein synthesis is exacerbated in obese inactive individuals. J Clin Endocrinol Metab 102(9): 3335-3345.

25. Dahlmans D, Houzelle A, Schrauwen P (2016) Mitochondrial dynamics, quality control and mRNA regulation in skeletal muscle: implications for obesity and related metabolic disease. Clin Sci 130(1): 843-852.

26. Strasser B (2013) Physical activity in obesity and metabolic syndrome. Ann N Y Acad Sci 1281: 141-159.

27. Vettor R, Miln G, Franzin C (2009) The origin of intermuscular adipose tissue and its pathophysiological implications. Am J Physiol Endocrinol Metab 297(5): E987-998.

28. Scarda A, Franzin C, Milan G (2010) Increased adipogenic conversion of muscle satellite cells in obese Zucker rats. Int J Obes 34(8): 13191327.

29. Thornell LE (2011) Sarcopenic obesity: satellite cells in the aging muscle. Curr Opin Clin Nutr Metab Care 14(1): 22-27.

30. Biolo G, Agostini F, Simanic B (2008) Positive energy balance is associated with accelerated muscle atrophy and increased glutathione turnover during 5 wk of bed rest. Am J Clin Nutr 88(4): 950-958.

31. Kang C, Ji LL (2013) Muscle immobilization and remobilization downregulates PGC-1-alpha signaling and the mitochondrial biogenesis pathway. J Appl Physiol 115(11): 1618-1625.

32. Vlietstra L, Meridith-Jones K, Steffings S (2017) Sarcopenic obesity is more prevalent in osteoarthritis than rheumatoid arthritis: are different processes involved? Rheumatology (Oxf) 56(10): 1816-1818.

33. Misra D, Fielding RA, Felson DT (2018) Risk of knee OA with obesity, sarcopenic obesity and sarcopenia. Arthritis Rheumatol.

34. Cruz-Jentoft AJ, Kieswetter E, Drey M (2007) Nutrition, frailty and sarcopenia. Agng Clin Exp Res 29(1): 43-48

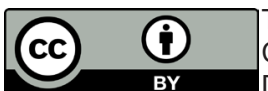

This work is licensed under Creative Commons Attribution 4.0 License

DOI: 10.19080/JPFMTS.2018.05.555675
35. Gale CR, Baylis D, Cooper C (2013) Inflammatory markers and incident frailty in men and women: the English Longtudinal Study of Ageing. Age (Dordr) 35(6): 2493-2501.

36. Valdes AM, Stocks I (2018) Osteoarthritis and ageing. EMJ 31: 116123.

37. Godziuk K, Prado CM, Woodhuse LJ (2018) The impact of sarcopenic obesity on knee and hip osteoarthritis a scoping review. BMC Muculoskelet. Disord 19: 271.

38. Ma J, Hwang SJ, McMahon GM (2016) Mid-adulthood cardiometabolic risk factor profiles of sarcopenic obesity. Obesity 24(2): 526-534.

39. Pasco JA, Sui SX, Tembo MC (2017) Sarcopenic Obesity and Falls in the Elderly. J Gerontol Geriatr Res 7: 2.

40. Tolea MI, Chrisphonte S, Galvin JE (2018) Sarcopenic obesity and cognitive performance. Clin Interv Aging 13: 1111-1119.

41. Zamboni M, Mazzali G, Fautin F (2008) Sarcopenc obesity: a new category of obesity in the elderly.

Nutr Metab Cardiovasc Dis 18(5): 388-395.

42. Lemos, Gallagher D (2017) Current body composition measurements techniques. Curr Opin Endocrinol Diab Obes 24(5): 310-314.

43. Bosy-Westphal A, Muller MJ (2015) Assessment of fat and lean body mass by quantitative magnetic resonance: a future technology of body composition research? Curr Opin Cln Nutr Metab Care 18(5): 446-451.

44. Bosy-Westphal A, Jensen B, Braun W (2017) Quantification of wholebody and segmental skeletal muscle mass using phase sensitive 8-electrode medical bioelectrical impedance devices. Eur J Clin Nutr 71(9): 1061-1067.

45. Fiatarone MA, Marks EC, Ryan ND (1990) High-intensity strength training in nonagenarians; Effects on skeletal muscle. JAMA 263(22): 3029-3034.

46. Naafs MA (2018) Selective Androgen Receptor Modulators (SARMs): A Mini-Review. Open Acc J Repro \& Sexual Disord 1(1): OAJRSD. MS.ID.000103.

\section{Your next submission with Juniper Publishers} will reach you the below assets

- Quality Editorial service

- Swift Peer Review

- Reprints availability

- E-prints Service

- Manuscript Podcast for convenient understanding

- Global attainment for your research

- Manuscript accessibility in different formats

( Pdf, E-pub, Full Text, Audio)

- Unceasing customer service

Track the below URL for one-step submission

https://juniperpublishers.com/online-submission.php 